\title{
Forum: «Médecine et économie: mariage de raison?»
}

\author{
9 février 2006, Centre de Congrès de Montreux
}

Dr. Philippe Freiburghaus, Président de la Société médicale de la Suisse romande

Le maintien de la qualité des soins et la meilleure maîtrise des coûts passent par une collaboration plus étroite des divers partenaires du système de santé. Convaincu de cette nécessité, le Groupe Mutuel a initié une démarche à la fois originale et ambitieuse auprès du monde médical des cantons romands et de Berne. Ce projet intitulé «Médecin Partenaire» a reçu un accueil favorable des différentes sociétés médicales de ces différents cantons.

Basé sur le dialogue et la concertation, ce projet propose une nouvelle approche pour trouver des pistes afin d'améliorer l'efficience médicale tout en maîtrisant mieux les coûts de la santé. Il s'est pour le moment limité à des rencontres individuelles avec les différents médecins partenaires et l'analyse de leurs statistiques. Mais, cela n'est qu'un premier pas qui laisse bien augurer des développements futurs.

L'un des fruits de cette collaboration entre un assureur et les professionnels de la santé, est la mise sur pied conjointe par la Société médicale de Suisse romande et le Groupe Mutuel d'un forum sous le thème «Médecine et économie: mariage de raison?». Il faut relever que c'est l'une des premières fois en Suisse qu'un assureurmaladie et une société médicale organisent ensemble une telle manifestation.

Pour donner une véritable plus-value à ce forum, il a semblé important aux organisateurs de faire intervenir des personnalités étrangères reconnues sur le plan européen dans le domaine de la santé, aux côtés de responsables et spécialistes de notre pays. Ceci devrait permettre d'apporter à la fois un regard neutre sur le système de santé suisse et une vision nouvelle ou pour le moins quelque peu différente des problèmes auxquels nous sommes confrontés. Les conférenciers aborderont plusieurs thèmes qui ont trait à la relation médecins-payeurs, à la formation des médecins, à l'environnement de la pratique médicale de demain, mais également aux rapports entre médecins et assureurs dans un contexte de responsabilités partagées. Ces quatre conférences s'inscrivent parfaitement dans les préoccupations actuelles de tous les acteurs du monde de la santé. Enfin, pour laisser la possibilité à tout un chacun d'exprimer son point de vue et d'apporter ses idées, il sera organisé durant l'après-midi huit ateliers sur diverses problématiques. Selon le vœu des organisateurs, ces réflexions partagées permettront de trouver des pistes et des solutions communes pour le futur.

Ce forum aura lieu le 9 février 2006, au Centre des congrès de Montreux. Inscription d'ici au 20 décembre sur www.groupemutuel.ch/ forum 2006 . 